\title{
A major-capsid-protein-based multiplex PCR assay for rapid identification of selected virulent bacteriophage types
}

\author{
Yannick Born ${ }^{1} \cdot$ Leandra E. Knecht ${ }^{1,2} \cdot$ Mirjam Eigenmann $^{1} \cdot$ Michel Bolliger $^{1} \cdot$ Jochen Klumpp $^{2} \cdot$ Lars Fieseler $^{1}(\mathbb{C}$
}

Received: 27 September 2018 / Accepted: 14 December 2018 / Published online: 23 January 2019

(c) The Author(s) 2019

\begin{abstract}
Bacteriophages represent a promising alternative for controlling pathogenic bacteria. They are ubiquitous in the environment, and their isolation is usually simple and fast. However, not every phage is suitable for biocontrol applications. It must be virulent (i.e., strictly lytic), non-transducing, and safe. We have developed a method for identifying selected types of virulent phages at an early stage of the isolation process to simplify the search for suitable candidates. Using the major capsid protein (MCP) as a phylogenetic marker, we designed degenerate primers for the identification of Felix O1-, GJ1-, N4-, SP6-, T4-, T7-, and Vi1-like phages in multiplex PCR setups with single phage plaques as templates. Performance of the MCP PCR assay was evaluated with a set of 26 well-characterized phages. Neither false-positive nor false-negative results were obtained. In addition, 154 phages from enrichment cultures from various environmental samples were subjected to MCP PCR analysis. Eight of them, specific for Salmonella enterica, Escherichia coli, or Erwinia amylovora, belonged to one of the selected phage types. Their PCR-based identification was successfully confirmed by pulsed-field gel electrophoresis of the phage genomes, electron microscopy, and sequencing of the amplified $m c p$ gene fragment. The MCP PCR assay was shown to be a simple method for preliminary assignment of new phages to a certain group and thus to identify candidates for biocontrol immediately after their isolation. Given that sufficient sequence data are available, this method can be extended to any phage group of interest.
\end{abstract}

\section{Introduction}

Bacteriophages (phages) are viruses that infect only bacteria. Due to the rise of antibiotic resistance in many relevant bacterial pathogens, interest in phage research is steadily increasing. Because of their unique properties, phages are promising alternatives for the control of bacteria. The major advantage is their host specificity, which enables a targeted treatment of a pathogen. In addition, phages are self-limiting and natural [1]. However, not every phage is suited

Handling Editor: Tim Skern.

Electronic supplementary material The online version of this article (https://doi.org/10.1007/s00705-019-04148-6) contains supplementary material, which is available to authorized users.

Lars Fieseler

lars.fieseler@zhaw.ch

1 Institute of Food and Beverage Innovation, Zurich University of Applied Sciences, 8820 Wädenswil, Switzerland

2 Institute of Food, Nutrition and Health, ETH Zurich, Zurich, Switzerland for application as a biocontrol agent. In general, biocontrol phages need to be virulent (i.e., strictly lytic) and non-transducing, and they should not carry genes known to encode toxins. They also must be stable during storage and application. Finally, they need to be propagatable to high titers on non-pathogenic production strains [2]. Phage biocontrol has been successfully applied in food pre- and postharvest to control the major foodborne pathogens Salmonella enterica, shiga-toxin-producing E. coli (STEC), Campylobacter jejuni, Listeria monocytogenes, and Staphylococcus aureus [2]. Likewise, phages have been used to control plant infections caused by Xanthomonas spp. or Erwinia amylovora [3], and they have been applied in aquaculture to treat bacterial infections of fish and shellfish [4].

The total number of phages on Earth is enormous and exceeds an estimated $10^{31}$ virions [5, 6]. Given this vast number, it is likely that there is a phage specific for any host that meets all the demands on a biocontrol phage. In general, phages can be easily isolated from environmental samples. Protocols for isolation of tailed phages (members of the order Caudovirales) typically involve an enrichment step followed by plating using the soft-agar overlay method 
$[7,8]$. Standard enrichments are usually performed with a single bacterial strain, while protocols adjusted towards isolation of polyvalent phages include sequential enrichment with several host strains [7,9]. Both methods normally yield numerous isolates. However, the subsequent characterization of all isolates to avoid redundancy and to identify virulent candidates is time consuming and laborious.

The increase in the number of completely sequenced phage genomes in the past years has enabled comparisons of whole genome sequences, which has revealed evolutionary relationships among different phage groups. Although clearer parameters to define genera, subfamilies, and families still need to be established, new phage subfamilies and genera have been proposed on the basis of molecular relationships (e.g., phages with $40 \%$ homologous proteins are members of the same genus) [10]. Importantly, members of the same subfamily or genus usually share a lifestyle and approximate genome size [11]. For example, a group of strictly lytic phages is formed by myoviruses related to Salmonella phage Felix O1 (FO1) with genomes of ca. $85-90 \mathrm{~kb}$ in size. They are classified as members of the subfamily Ounavirinae [12]. The FO1-like Salmonella phages Mushroom and FO1a are components of commercially available phage cocktails (IntestiPhage ${ }^{\mathrm{TM}}$ and PhageGuard $\mathrm{S}^{\mathrm{TM}}$, respectively) $[13,14]$. GJ1-like phages are also virulent myoviruses. $\Phi$ EcoM-GJ1 infects enterotoxigenic E. coli O149:H10:F4 strains [15]. Other phages related to GJ1, such as the E. amylovora phage vB_EamM-Y2 or Pectobacterium carotovorum phage $\mathrm{PM} 1$ have similar genome sizes of ca. $55 \mathrm{~kb}$ as well as broad host ranges and have been shown to effectively limit the growth of their target bacteria in vitro as well as in vivo [16, 17]. The strictly lytic N4-like phages belong to the family Podoviridae and have genomes of approximately 70-75 kb in size [18]. The N4-like Escherichia phage EC1-UPM reduces the severity of colibacillosis in chickens caused by E. coli O78:K80 strains [19]. SP6like phages are virulent podoviruses of one genus (Sp6virus) with genomes of ca. $45 \mathrm{~kb}$ in size [20]. The SP6-like Salmonella phage UAB_Phi78, which displays a broad host range on strains of Salmonella enterica subsp. enterica serovars Typhimurium and Enteritidis, was part of a cocktail that greatly reduced Salmonella contamination on various food surfaces [21]. The relatively small and strictly lytic T7-like viruses (ca. $40 \mathrm{~kb}$ ) also belong to one genus (T7virus) in the family Podoviridae. At their tail spikes, many T7-like phages have enzymes that hydrolyze bacterial exopolysaccharides, which assist in infection of the host [22-24]. The safety of the virulent T4-like myoviruses from the subfamily Tevenvirinae was demonstrated by oral application of phage preparations to healthy children and adults. No adverse effects were reported $[25,26]$. A prominent member of the T4-like phages is Salmonella phage vB_SenM-S16, which possesses an extraordinarily broad host range [27]. Finally, the virulent Vi1-like phages share a myovirus morphology, with genomes of ca. 155-160 kb in size, and they belong to the new family Ackermannviridae [28, 29]. The Vi1-like phage LIMEstone1 has been shown to significantly reduce the incidence and severity of soft rot on potato tubers caused by Dickeya solani [30]. Likewise, contamination with $E$. coli O157:H7 on various foods has been shown to be reduced by EcoShield ${ }^{\mathrm{TM}}$, a commercial product composed of three phages, one of which (ECML-4) is a member of the genus Vilvirus [31, 32]. However, some Vil-like phages have been reported to be generalized transducers [33] - a characteristic that is incompatible with its use in phage biocontrol and needs to be clarified beforehand.

The established groups of FO1-, GJ1-, N4-, SP6-, T4-, T7-, and Vi1-like phages all comprise strictly lytic phages, and representatives of each group have been successfully applied to control pathogenic bacteria. An early assignment of a new phage to one of these groups could therefore enable preselection to accelerate phage isolation and characterization. Genome sequencing allows identification of the phylogenetic relationships of a new isolate, but it is still associated with high costs and complex data analysis. In order to circumvent the need for complete sequencing, molecular markers (signature genes) could be studied instead. These markers should be universally encoded by all tailed phages, and, more importantly, the phylogenetic groups formed by comparisons of whole genome sequences and the corresponding markers need to be identical. There are several candidates for such signature genes, and their usefulness as molecular markers has already been demonstrated. For example, the grouping of Vil-like phages has been corroborated by phylogenetic analysis of single signature genes (major capsid protein [MCP], DNA polymerase, DNA ligase, terminase large subunit) [29]. A phylogenetic tree of the former subfamily "Felixounavirinae" constructed by analysis of concatenated protein sequences (MCP, tail sheath protein, portal protein) also confirmed clustering based on whole-genome comparisons [34]. Signature genes have also been used to study viral diversity in environmental samples. For example, the diversity of T7-like cyanoviruses has been analyzed with the help of three signature genes (DNA polymerase, MCP, photosynthesis gene $p s b A$ ), and these viruses have been specifically isolated using degenerate primers targeting the DNA polymerase gene [35]. In a comprehensive study of phages infecting enterobacteria, Grose and Casjens [11] showed that the chance of assigning a new isolate to the correct cluster by its MCP sequence is as high as $96.4 \%$. Hence, a new phage isolate should be correctly grouped after MCP sequence analysis. Similar correlation values of $97.6 \%$ and $98.8 \%$ have been reported for the tail tape measure protein (TMP) $[11,36]$. However, TMP cannot be used to classify members of the family Podoviridae because they do not encode the protein. 
In this study, we used the major capsid protein as a molecular marker and designed degenerate primers to specifically identify members of the FO1-, GJ1-, N4-, SP6-, T4-, T7-, and Vi1-like phage groups early during the isolation process. We determined sensitivity and specificity of the newly developed MCP PCR assays and, as a proof of concept, isolated and identified phages infecting E. coli, S. enterica, and E. amylovora. Their presumptive grouping based on MCP PCR results was confirmed by electron microscopy (EM), pulsed-field gel electrophoresis (PFGE), and sequencing of the $m c p$ gene fragment. The results demonstrate that the MCP PCR assay is a convenient and reliable tool for the quick identification of selected phages.

\section{Materials and methods}

\section{Primer design}

MCP amino acid sequences of the "type phages" T4, T7, N4, ФEcoM-GJ1 (GJ1), SP6, Felix O1 (FO1), and ViI, and of a diverse set of closely related phages (Table 1) were used for the design of degenerate MCP primers (Table 2)
Table 1 Phages used for primer design

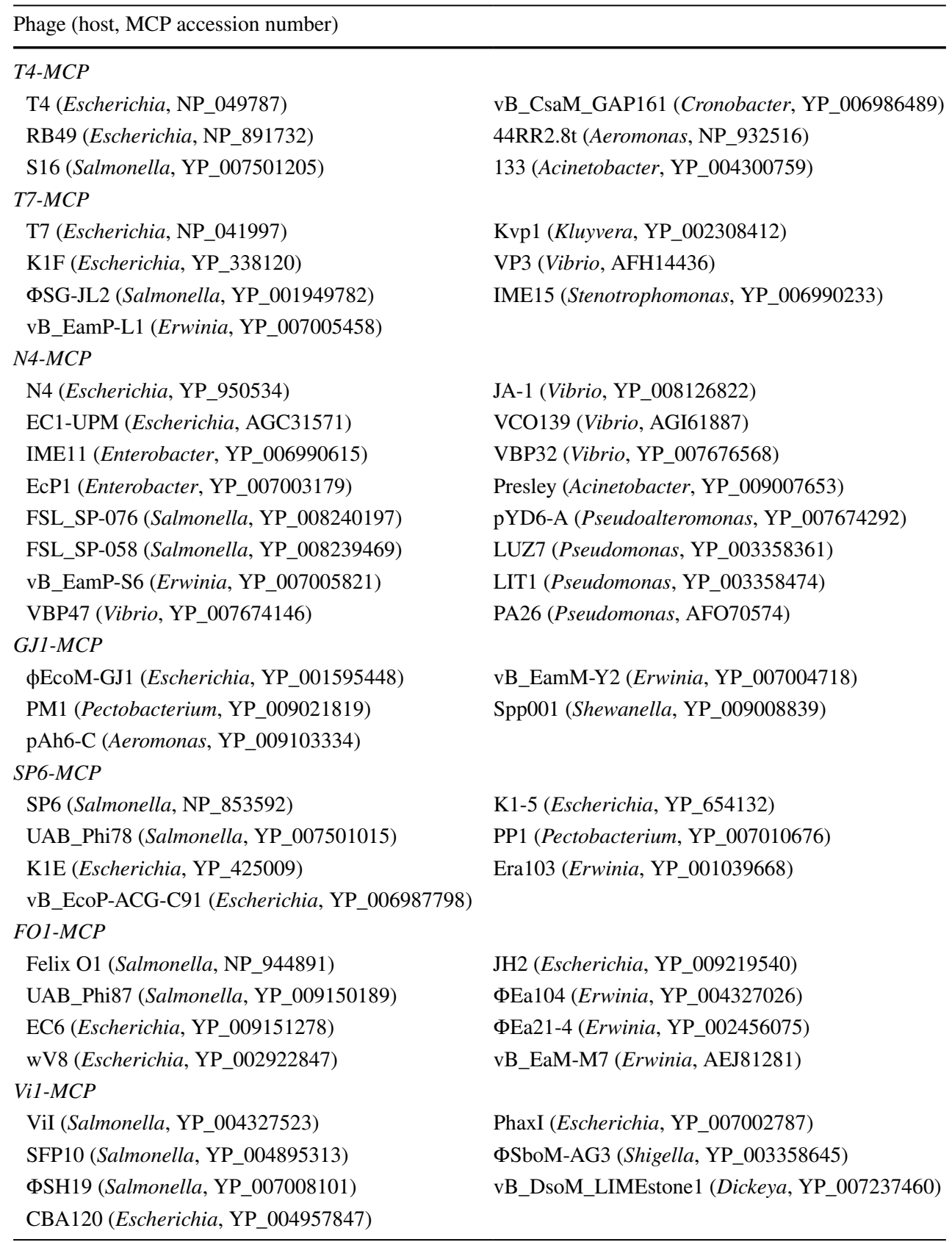


Table 2 Primers designed and used in this study

\begin{tabular}{llc}
\hline Name & Sequence $\left(5^{\prime} \rightarrow 3^{\prime}\right)$ & $\begin{array}{l}\text { Degen- } \\
\text { eracy } \\
\text { score }\end{array}$ \\
\hline T4-fw & CCC TGC TGT TCC AGA TCG ANA ARG ARG C & 16 \\
T4-rev & CTG CCT GGC GTA CTG GTC DAT RWA NAC & 48 \\
T7-fw & GAC AAG CGG AAG GAC ATC AAN CAY ACN GAR A & 64 \\
T7-rev & CGC GTA GTT GGC GGC RTT NGG CAT NA & 32 \\
N4-fw & GGA TGA TCG TAA TAT TAA TGA TCA GGG NAT HRA YGC & 48 \\
N4-rev & GAC ATA AAG CCC ATT TCG CCR WAN GGR TC & 32 \\
GJ1-fw & GGC TGC GCG TAT GAT TAG GAY ATH GAY GA & 12 \\
GJ1-rev & CCA ATG CAT CAC CGG CAD CCA DAT YTC & 18 \\
SP6-fw & CAC CGT GAT TGC GCG TAA YAC NGT NGC & 32 \\
SP6-rev & TTC CCA ACG ATC CGG AAT NGC NCC YTC & 32 \\
FO1-fw & CGC CAT TGA AGA ACT GCG TRW RCA YAT GGA & 16 \\
FO1-rev & GGC ATC ATA TAG GAA TGC GCY TCR AAR TC & 8 \\
Vi1-fw & GCC GAT TAA TAT TGC GAT GGA YTT YTT & 4 \\
Vi1-rev & CCA GCA TAA AGG TCA TAA ATT TCC AYT TYT C & 4 \\
\hline
\end{tabular}

applying the Codehop strategy [37]. Primer pairs with the lowest degeneracy scores possible were selected. All of the phages used for the design of the T4-MCP primers belonged to the T4 supercluster infecting enterobacteria presented by Grose and Casjens [11], except for phage 133, which is specific for Acinetobacter. They are all members of different genera of the subfamily Tevenvirinae. The T7-MCP primers were based on MCP sequences of a few phages infecting various species of the gammaproteobacteria, all of which are members of the T7-like cluster of the T7-supercluster. Likewise, SP6-like phages were selected from the SP6cluster of the T7-supercluster [11]. Oligonucleotides for the specific detection of N4-like phages were designed based on MCP sequences of N4-like phages as published by Chan et al. [38]. Sequences of phages infecting bacteria other than gammaproteobacteria had to be excluded due to insufficient sequence similarity. The GJ1-specific primers were designed based on MCP sequences of members of the GJ1-like cluster [11] extended by Shewanella phage Spp001 [39] and Aeromonas phage pAh6-C [40]. The FO1 primer pair was designed based on the MCP sequences of the members of the Felix $\mathrm{O} 1$ cluster published by Grose and Casjens [11], except for FO1a, whose MCP sequence is identical to that of Felix O1. All of them belong to the subfamily Ounavirinae [12]. Finally, Vi1-like phages were selected based on their membership in the former genus "Viunalikevirus" suggested by Adriaenssens et al. [29].

\section{Dotplot and phylogenetic analysis}

Dotplot analysis of MCP sequences used for primer design was performed with Gepard using standard parameters [41]. A phylogenetic tree was constructed with CLC Main
Workbench (version 7.9.1, QIAGEN Bioinformatics, Aarhus, Denmark), applying the neighbor-joining algorithm and bootstrap analysis. Partial $m c p$ gene sequences of the new isolates were included to study their phylogenetic relationships.

\section{Preparation of templates (plaque PCR)}

Phages were cultivated using the soft-agar overlay method [8] with temperatures and media listed in Table S1. Wellseparated single plaques were picked with a Pasteur pipette, and the agar plugs were transferred to PCR test tubes containing $100 \mu \mathrm{l}$ of $\mathrm{SM}$ buffer $(50 \mathrm{mM}$ Tris, $100 \mathrm{mM} \mathrm{NaCl}$, $8 \mathrm{mM} \mathrm{MgSO}_{4}, \mathrm{pH}$ 7.4). The tubes were incubated for $1 \mathrm{~h}$ at room temperature to allow diffusion of the phages into the buffer. Aliquots of $50 \mu \mathrm{l}$ were removed for any subsequent analysis requiring intact virus particles, and the remaining $50 \mu \mathrm{l}$ was heated in a thermocycler for $10 \mathrm{~min}$ at $95^{\circ} \mathrm{C}$ and cooled down to $8^{\circ} \mathrm{C}$. This caused melting of the agar plug and release of the phage particles into the buffer. Two microliters of the heat-treated samples was used as template for the PCR assay.

\section{PCR conditions}

Phages T4, T7, and N4 were used as positive controls for the respective PCRs. The positive controls of the other PCRs were Salmonella phage FO1-E2 (FO1-specific PCR; Marti et al., unpublished), Erwinia phage vB_EamP-S2 (SP6; [17, 42]), Erwinia phage vB_EamM-Y2 (GJ1; [17]), and Salmonella phage KCK6 (Vi1; Born et al., unpublished). KAPA Taq ReadyMix (Sigma-Aldrich, Buchs, Switzerland) was used for PCR following the manufacturer's instructions. 
Reactions were performed in $20-\mu$ l volumes $(10 \mu \mathrm{l}$ of KAPA Taq, $0.8 \mu \mathrm{l}$ of each primer $[10 \mu \mathrm{M}], 6.4 \mu \mathrm{l}$ of $\mathrm{ddH}_{2} \mathrm{O}$, and $2 \mu \mathrm{l}$ of template) with an annealing temperature of $50{ }^{\circ} \mathrm{C}(\mathrm{T} 4$, FO1, GJ1, Vi1) or $55^{\circ} \mathrm{C}(\mathrm{N} 4, \mathrm{SP} 6, \mathrm{~T} 7)$.

\section{Detection limits}

For determination of the detection limits, plaques of the respective positive controls were picked and resuspended in $100 \mu \mathrm{l}$ of SM buffer as described above. After $1 \mathrm{~h}$, phages were serially diluted, and the concentrations of infectious virions were determined using soft-agar overlays. Immediately thereafter, the same dilution series was heated to $95{ }^{\circ} \mathrm{C}$ for $10 \mathrm{~min}$ and used as a template for PCR. These experiments were independently performed twice.

\section{Multiplex PCR}

Reactions generated positive results with annealing temperatures over a wide range. To increase the throughput of the method, screening was performed in multiplex PCRs. MCP PCR format with similar annealing temperatures but generating fragments of different sizes that allowed clear visual discrimination after gel electrophoresis were combined. Conditions were established to run triplex PCRs in 20- $\mu$ l volumes $(10 \mu \mathrm{l}$ of KAPA Taq, $0.8 \mu \mathrm{l}$ of each primer $(10 \mu \mathrm{M})$, $3.2 \mu \mathrm{l}$ of $\mathrm{ddH}_{2} \mathrm{O}$, and $2 \mu \mathrm{l}$ of template). T4/T7/SP6 (annealing temperature: $52{ }^{\circ} \mathrm{C}$ ) and N4/FO1/GJ1 $\left(54{ }^{\circ} \mathrm{C}\right)$ were successfully combined, whereas SP6 could also be replaced by Vi1. Detection limits were not determined, but picking single plaques and running the PCRs according to the standard protocol was sufficient to generate positive results.

\section{Specificity/sensitivity testing}

A set of 26 phages (Table 3) were tested to evaluate the sensitivity and specificity of the method. Phages were cultivated (Table S1) and picked as described above. PCRs were performed in single reactions and repeated once.

\section{Phage isolation}

Phages were isolated from various environmental samples (ponds, sewage water, soil, and animal feces). Solid samples were mixed with $10 \mathrm{ml}$ of SM buffer per gram and homogenized. Solid particles were removed by centrifugation. Enrichment was performed in volumes of $20 \mathrm{ml}$ and contained $10 \mathrm{ml}$ of $2 \times$ concentrated LB broth, $2 \mathrm{ml}$ of SM buffer $(10 \times), 2 \mathrm{ml}$ of a bacterial overnight culture, and $6 \mathrm{ml}$ of the preprocessed environmental sample. After overnight incubation at the desired temperature, bacteria and other particles were removed by centrifugation $(2 \mathrm{~min}, 10,000 \times g)$, and the supernatants were sterilized using $0.2-\mu \mathrm{m}$-pore-size filters. To obtain single plaques, dilution series were plated using the soft-agar overlay method [8]. Incubation temperatures and media used to propagate the new phage isolates are listed in Table S1. Single plaques were then analyzed by multiplex MCP PCR. Positive results were confirmed by PCR using the corresponding primer pair only.

\section{Phage purification and characterization}

MCP-PCR-positive phages were propagated and purified using $\mathrm{CsCl}$ density gradient centrifugation as described elsewhere [17, 43]. Electron microscopy of negatively stained phage particles was performed as described earlier [44]. For the extraction of phage DNA, CsCl-purified virus particles were dialyzed against a 1,000-fold excess of SM buffer $(6 \mathrm{~h}, \mathrm{RT})$. Free nucleic acids were degraded by treatment for 15 min at $37{ }^{\circ} \mathrm{C}$ with DNase I (final concentration: $1 \mathrm{U} / \mathrm{ml}$ ) and RNase A $(50 \mu \mathrm{g} / \mathrm{ml})$. Nucleases were inactivated and phage capsids were degraded by treatment for $1 \mathrm{~h}$ at $56^{\circ} \mathrm{C}$ with EDTA (20 mM, pH 8.0), proteinase K (50 $\mu \mathrm{g} / \mathrm{ml})$, and SDS $(0.5 \%$ [wt/vol] ), and DNA was purified using a phenolchloroform extraction procedure with subsequent ethanol precipitation [43]. Genome sizes were determined by PFGE (settings: $6 \mathrm{~V} / \mathrm{cm}$, switch times: $2-25 \mathrm{~s}, 20 \mathrm{~h}, 14^{\circ} \mathrm{C}$ ) using a Chef DR III apparatus (Bio-Rad, Reinach, Switzerland).

\section{Results}

\section{Distinct clusters revealed by MCP sequence comparisons}

The MCP sequences that were used for the primer design were compared by dotplot analysis (Fig. 1). The seven phage types studied formed distinct clusters with clear similarities only within each group. Subclusters were observed in the groups of the N4-, GJ1-, and FO1-like phages. There were two subclusters in the GJ1 and FO1 groups, which correlated well with the host specificities of the corresponding phages. Phages infecting enterobacteria were separate from those infecting non-enterobacteria and such specific for Escherichia and Salmonella were separate from the Erwinia phages. Similar tendencies were observed for the N4-like phages. However, the subclusters were not as distinct. These clear clusters were also prominent in the phylogenetic tree (Fig. 2).

\section{Direct analysis of plaques by MCP PCR}

The detection limit was defined as the lowest number of PFUs needed to produce a visible band in gel electrophoresis after PCR. The limits ranged from $10^{1}$ to $10^{3}$ PFU per reaction, which is equivalent to using a template concentration 
Table 3 Complete list of phages used for performance testing

\begin{tabular}{|c|c|c|c|c|c|c|c|}
\hline Name & Family $^{\mathrm{a}}$ & Subfamily & Genus & Host & Lifestyle $^{b}$ & Genome size $(\mathrm{kb})$ & Reference \\
\hline vB_EamP-L1 & $P$ & Autographivirinae & T7virus & Erwinia & $\mathrm{v}$ & 39.3 & [17] \\
\hline $\mathrm{T} 7$ & $P$ & Autographivirinae & T7virus & Escherichia & $\mathrm{v}$ & 39.9 & $\mathrm{DSMZ}^{\mathrm{c}}$ \\
\hline vB_EamP-S2 & $P$ & Autographivirinae & SP6virus & Erwinia & $\mathrm{v}$ & 45.5 & {$[17,42]$} \\
\hline N4 & $P$ & - & N4virus & Escherichia & $\mathrm{v}$ & 70.2 & [18] \\
\hline vB_EamP-S6 & $P$ & - & - & Erwinia & $\mathrm{v}$ & 74.7 & [17] \\
\hline$\varphi 29$ & $P$ & Picovirinae & Phi29virus & Bacillus & $\mathrm{v}$ & 19.3 & {$[63]$} \\
\hline $\mathrm{P} 22$ & $P$ & - & P22virus & Salmonella & $\mathrm{t}$ & 41.7 & DSMZ \\
\hline vB_EamM-Y2 & $M$ & - & - & Erwinia & $\mathrm{v}$ & 56.6 & {$[17]$} \\
\hline vB_EamM-M7 & $M$ & Ounavirinae & Ea214virus & Erwinia & $\mathrm{v}$ & 84.7 & [17] \\
\hline FO1-E2 & $M$ & Ounavirinae & Felixolvirus & Salmonella & $\mathrm{v}$ & 83.3 & Marti et al. ${ }^{\mathrm{d}}$ \\
\hline JG004 & $M$ & - & Pakpunavirus & Pseudomonas & $\mathrm{v}$ & 93.0 & {$[64]$} \\
\hline S16 & $M$ & Tevenvirinae & S16virus & Salmonella & $\mathrm{v}$ & 160.2 & {$[27]$} \\
\hline $\mathrm{T} 4$ & $M$ & Tevenvirinae & T4virus & Escherichia & $\mathrm{v}$ & 168.9 & DSMZ \\
\hline LBL3 & $M$ & - & Pbunavirus & Pseudomonas & $\mathrm{v}$ & 64.4 & {$[65]$} \\
\hline A511 & $M$ & Spounavirinae & P100virus & Listeria & $\mathrm{v}$ & 137.6 & {$[44,66]$} \\
\hline P100 & $M$ & Spounavirinae & P100virus & Listeria & $\mathrm{v}$ & 131.4 & {$[66,67]$} \\
\hline K & $M$ & Spounavirinae & Kayvirus & Staphylococcus & $\mathrm{v}$ & 148.3 & {$[66,68,69]$} \\
\hline $\mathrm{P} 2$ & $M$ & Peduovirinae & P2virus & Escherichia & $\mathrm{t}$ & 33.6 & {$[70]$} \\
\hline TK611 & $S$ & - & T5virus & Salmonella & $\mathrm{v}$ & 120.9 & Born et al. ${ }^{\mathrm{d}}$ \\
\hline$\lambda$ & $S$ & - & Lambdavirus & Escherichia & $\mathrm{t}$ & 48.5 & DSMZ \\
\hline P35 & $S$ & - & - & Listeria & $\mathrm{v}$ & 35.8 & [71] \\
\hline P40 & $S$ & - & - & Listeria & $\mathrm{v}$ & 35.6 & [71] \\
\hline P70 & $S$ & - & P70virus & Listeria & $\mathrm{v}$ & 67.2 & [72] \\
\hline A500 & $S$ & - & - & Listeria & $\mathrm{t}$ & 38.9 & [72] \\
\hline A118 & $S$ & - & - & Listeria & $\mathrm{t}$ & 40.8 & [73] \\
\hline KCK6 & $A$ & Cvivirinae & Vilvirus & Salmonella & $\mathrm{v}$ & 158.5 & Born et al. ${ }^{\mathrm{d}}$ \\
\hline
\end{tabular}

${ }^{\mathrm{a}} \mathrm{P}$, Podoviridae; $M$, Myoviridae; $S$, Siphoviridae; A, Ackermannviridae

${ }^{\mathrm{b}} \mathrm{v}$, virulent (strictly lytic); t, temperate

${ }^{c}$ DSMZ, Deutsche Sammlung von Mikroorganismen und Zellkulturen (German Collection of Microorganisms and Cell Culture)

dunpublished data

of $10^{4}$ to $10^{6} \mathrm{PFU} / \mathrm{ml}$. Since we used a tenfold dilution series without further gradation, the actual limits might have been slightly lower. Detection limits were not determined for the multiplex assays. Most importantly, the phage DNA concentrations in the picked and heated agar plugs were sufficient in all cases to generate positive PCR results. In the analysis of the 26 well-characterized phages, not a single false negative or false positive result was obtained (data not shown). Thus, both the specificity and the sensitivity of all primer pairs were $100 \%$. A few reactions resulted in nonspecific results, seen as faint bands that differed in size from the positive controls. We rated these as negative.

\section{Low environmental frequency of the targeted phage groups revealed by multiplex MCP PCR}

Salmonella, Escherichia, and Erwinia phages were isolated from different environmental samples. In total, 154 plaques were picked and analyzed. Of these, 14 belonged to one of the seven groups of interest based on MCP PCR analysis (Table 4). More precisely, Escherichia phage L2 was a presumptive T4-like isolate, while the T7-MCP PCR was positive when using phage L3 as a template. Salmonella phages MOE1 and MOE2 were potential N4-like phages. Erwinia phage QceA2 was found to be GJ1-positive. Escherichia phages PGP and VNV were presumptive FO1like phages, as were the other six positive Erwinia samples. Since the Erwinia samples were collected from the same quince orchard, they were likely to be identical. Thus, only QceB10 was selected, and we did not analyze the remaining FO1-positive Erwinia isolates. Salmonella phage DaiSi was a presumptive Vi1-like phage. The initial grouping of the new phage isolates was confirmed by estimating their genome sizes using PFGE and analysis of their morphologies by EM (except for L3, which we failed to propagate to high titers). The results are summarized in Table 4, while 


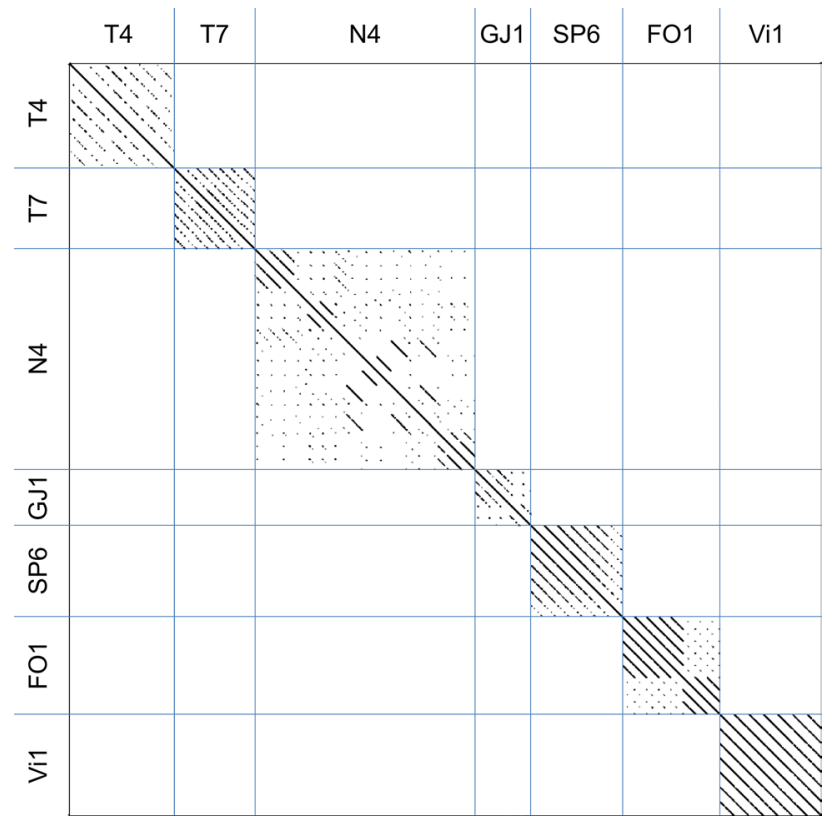

Fig. 1 Dotplot analysis of MCP sequences used for the primer design. The order of the phages is according to Table 1

the original data from PFGE analysis (Fig. S1) and electron micrographs (Fig. S2) are provided as supplementary material. In addition, PCR products of the nine selected phages were sequenced and compared by blastx [45] with the nonredundant GenBank database. The closest relative of the presumptive T4-like Escherichia phage L2 was found to be Escherichia phage vB_EcoM_VR26 (100\% amino acid sequence identity) [46], which belongs to the genus $S p 18 v i$ rus of the subfamily Tevenvirinae. L3 was most closely related to Escherichia phage LM33_P1 (100\%), a member of the genus T7virus [47]. The MCP sequences of the putative N4-like Salmonella phages MOE1 and MOE2 shared 99\% amino acid sequence identity with Salmonella phage FSL SP-058. FSL SP-058 was proposed to be grouped into the genus Sp58virus of N4-like viruses [18, 48]. Erwinia phage QceA2, which was isolated as a presumptive GJ1like phage, shared $99 \%$ amino acid sequence identity with Erwinia phage vB_EamM-Y2, which is closely related to phage $\Phi E c o M-G J 1[11,17]$. The presumptive FO1-like isolates PGP and VNV were found to be most similar to Salmonella phage UAB_Phi87 (99\%) and Escherichia phage vB_EcoM-VpaE1 (100\%), respectively. Both are members of the genus Felixolvirus in the subfamily Ounavirinae $[12,49,50]$. Erwinia phage vB_EamM-M7, the closest relative of the putative FO1-like Erwinia phage QceB10 (99\%), belongs to the same subfamily but to another genus (Ea214virus) [12]. The presumptive Vi1-like Salmonella phage DaiSi shared a 99\% amino acid sequence identity with Salmonella phage FSL SP-029, which is also related to ViI [48]. Phylogenetic analysis of the partial MCP sequences also confirmed classification of the novel phages into the expected groups (Fig. 2).

The partial MCP sequences of the isolated phages were deposited in the GenBank database under the accession numbers MK163343 (L2), MK163344 (L3), MK163345 (MOE1), MK163346 (MOE2), MK163347 (QceA2), MK163348 (PGP), MK163349 (VNV), MK163350 (QceB10), and MK163351 (DaiSi).

\section{Discussion}

Due to the enormous diversity of bacteriophages in nature, the search for new phages with high potential for biocontrol purposes is rather limited. A new phage isolate needs to fulfill important requirements, such as being strictly lytic, specific, safe, and non-transducing, if applied in biocontrol. In addition, as demonstrated here, the FO1-, GJ1-, N4-, SP6-, T4-, T7-, and Vi1-like phages seem to be present at only a low frequency in environmental samples. A comprehensive analysis of phages infecting enterobacteria showed that members of the same cluster share the same lifestyle [11]. A simple classification of new isolates similar to the $16 \mathrm{~S}$ rRNA analysis of bacteria could thus facilitate the identification of new candidates. The approach could be hampered by the mosaic architecture of phage genomes due to extensive horizontal gene transfer [5], which could lead to random isolation if classification relied on a single gene only. However, horizontal transfer of certain genes such as those encoding the head proteins is limited, because they interact in building essential structures. Random recombination events can lead to non-functional hybrids that are then lost from the population [51]. Consequently, genes encoding proteins such as the major capsid protein or the tail tape measure protein are conserved and can be used as signature genes for classification of phages $[11,36]$. In this study, we selected MCP as a molecular marker for early assignment of new phages to a certain group and thus for a preliminary identification of their lifestyle. There is a high correlation (96.4\%) between MCP types and whole-genome clusters of enterobacteria phages, as shown by Grose and Casjens [11]. Eleven of the twelve exceptions resided in the lambda supercluster. These exceptions are irrelevant for our primers, which were designed based on only strictly lytic phages. Also, there is no cluster that contains both strictly lytic and temperate phages, and no phage has recently switched between the two lifestyles [11].

None of the 26 phages with known classification gave false positive results in our performance testing. This collection was greatly expanded by our screening of environmental isolates. In every case, the closest relatives deduced from sequence comparisons were members of the expected groups. Genome sizes determined by PFGE further 


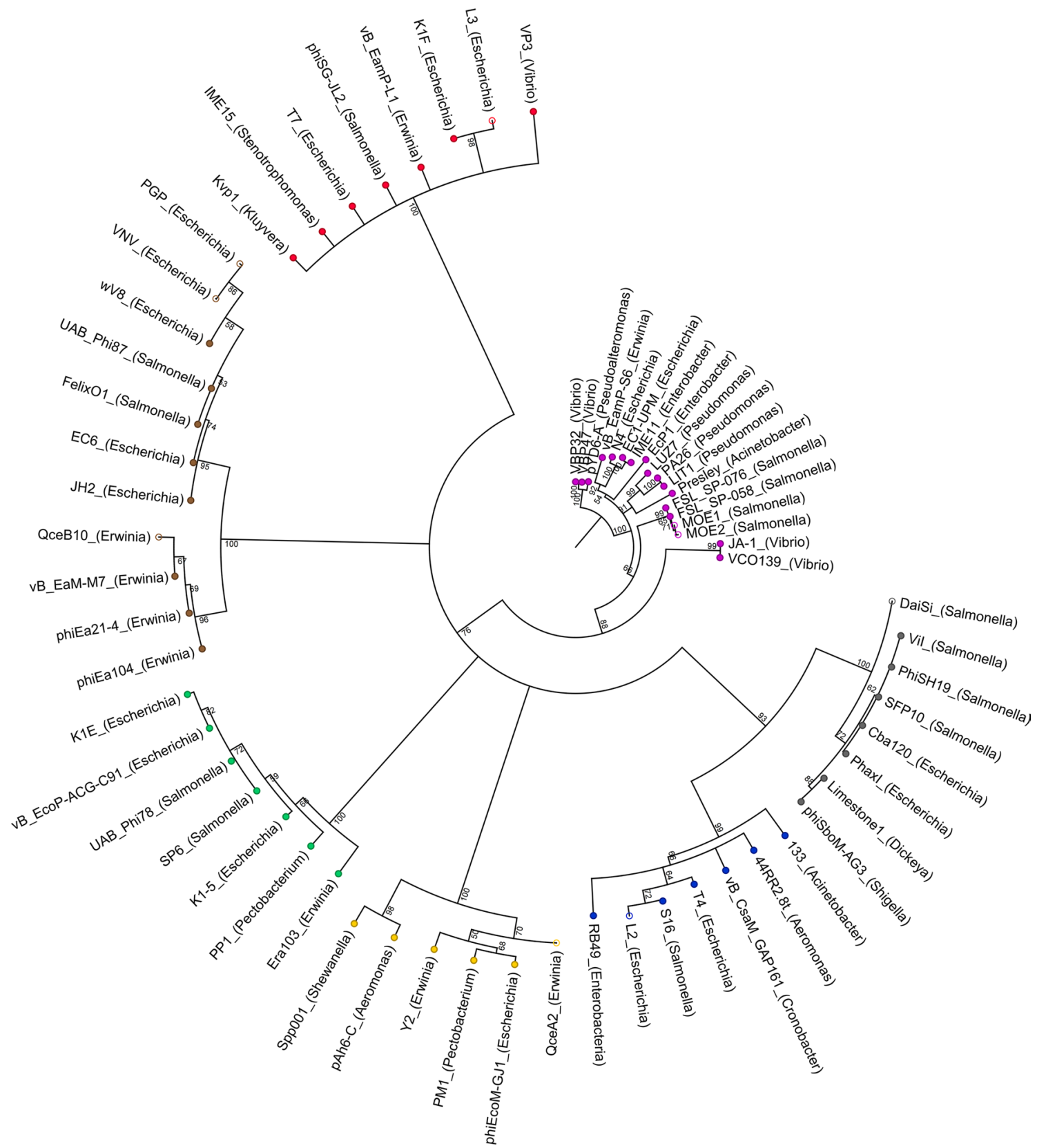

Fig. 2 Phylogenetic analysis of novel phages. New MCP sequences were aligned with those used for primer design. A neighbor-joining tree was constructed with 1,000 bootstrap replicates. Branches with

supported the initial identification, and EM-based analysis of the morphology of each isolate was in agreement with the MCP PCR prediction. We consider these analyses sufficient to confirm the presumptive MCP PCR results, since both genome sizes and tail types within clusters are uniform [11]. Thus, there were no false positive results, i.e., the specificity was $100 \%$. This finding further indicates that the degeneracy of the primers did not cause nonspecific annealing. False negatives, however, would not have been identified in the field study owing to the experimental setup. In theory, false values $<50 \%$ were collapsed. Nodes are colored according to phage groups. Purple, N4; grey, Vi1; blue, T4; yellow, GJ1; green, SP6; brown, FO1; red, T7. Open nodes indicate novel phages

negative results could originate from insufficient sequence similarity for primer binding, impurities, or concentrations below the detection limit. Lack of sensitivity is most probably not an issue for the new method presented here. Picking a plaque using a Pasteur pipette and resuspending it in $1 \mathrm{ml}$ of SM buffer usually yields a final concentration of $>10^{6} \mathrm{PFU} /$ $\mathrm{ml}$. The detection limits ranged from $10^{1}-10^{3} \mathrm{PFU}$ per reaction (equal to a required template concentration of $10^{4}-10^{6}$ $\mathrm{PFU} / \mathrm{ml}$ ), which is in the same range as the detection limits reported in other studies $[34,35]$. In fact, all positive control 
Table 4 Properties of isolated phages. Presumptive grouping is based on MCP PCR analysis. Genome sizes were determined by PFGE (Fig. S1); morphology was analyzed by electron microscopy (Fig. S2). nd: not determined

\begin{tabular}{|c|c|c|c|c|c|c|}
\hline \multirow[t]{2}{*}{ Isolate } & \multirow[t]{2}{*}{ Host } & \multirow{2}{*}{$\begin{array}{l}\text { Presumptive } \\
\text { grouping }\end{array}$} & \multicolumn{2}{|l|}{ Expected } & \multicolumn{2}{|c|}{ Experimental confirmation } \\
\hline & & & Genome size & Family $^{\mathrm{a}}$ & Genome size & Tail morphology \\
\hline L2 & E. coli & $\mathrm{T} 4$ & $160-175 \mathrm{~kb}$ & $M$ & $160 \mathrm{~kb}$ & contractile \\
\hline L3 & E. coli & $\mathrm{T} 7$ & $40 \mathrm{~kb}$ & $P$ & nd & nd \\
\hline MOE1 & S. Typhimurium & N4 & $70-75 \mathrm{~kb}$ & $P$ & $72 \mathrm{~kb}$ & short \\
\hline MOE2 & $S$. Typhimurium & N4 & $70-75 \mathrm{~kb}$ & $P$ & $72 \mathrm{~kb}$ & short \\
\hline QceA2 & E. amylovora & GJ1 & $55 \mathrm{~kb}$ & $M$ & $55 \mathrm{~kb}$ & contractile \\
\hline PGP & E. coli & FO1 & $85-90 \mathrm{~kb}$ & $M$ & $85 \mathrm{~kb}$ & contractile \\
\hline VNV & E. coli & FO1 & $85-90 \mathrm{~kb}$ & $M$ & $85 \mathrm{~kb}$ & contractile \\
\hline QceB10 & E. amylovora & FO1 & $85-90 \mathrm{~kb}$ & $M$ & $83 \mathrm{~kb}$ & contractile \\
\hline DaiSi & $S$. Typhimurium & Vi1 & $155-160 \mathrm{~kb}$ & $A$ & $158 \mathrm{~kb}$ & contractile \\
\hline
\end{tabular}

${ }^{a} P$, Podoviridae; $M$, Myoviridae; A, Ackermannviridae
PCRs performed with an undiluted template were clearly positive, demonstrating that picking a plaque and using it according to the presented protocol does not produce false negative results in either single or multiplex reactions.

All primers used in this study were designed based on sequences of virulent phages (Table 1). Depending on the current taxonomy of these phages (which is highly dynamic in some cases [52]), the specificity of each primer set is at a certain taxonomic level. Alignment of the MCP sequences of all members of the $\mathrm{T} 4$ cluster indicated conservation of certain regions [11]. The T4 MCP primers target such regions. Their design is based on a few phages of different genera of the subfamily Tevenvirinae, which comprises a constantly growing number of genera whose members are specific for gammaproteobacteria [10, 53]. The T4 MCP PCR assay is therefore expected to identify phages of the subfamily Tevenvirinae that infect gammaproteobacteria. Escherichia phage vB_EcoM_VR26, the closest relative of the new isolate L2, belongs to the genus Sp18virus of the subfamily Tevenvirinae [46].

T7 and SP6 are the prototype phages of their respective genera (T7virus and SP6virus). Both belong to the subfamily Autographivirinae [20]. The T7- and SP6-MCP PCRs are intended to detect phages at the genus level. The T7-MCP PCR could not amplify the SP6-prototype virus and vice versa, confirming the genus-specificity of these two MCP PCRs. Escherichia phage LM33_P1, which was found to be the closest relative of L3, is a member of the genus T7virus [47]. A SP6-like phage could not be isolated in our study.

It is difficult to clearly pinpoint the specificity to a certain taxon in the case of the N4-MCP PCR. The phages used for the design of our N4 primers belong to three genera of N4-like phages (G7cvirus, Lit1virus, Sp58virus), whereas only 67 cvirus belongs to the proposed subfamily "Enquartavirinae" [18]. The fourth proposed genus of N4-like phages, "Dss3virus", consisting of two viruses infecting Ruegeria spp. and Sulfitobacter spp., respectively, was not represented in our selection due to insufficient sequence similarity. Phage
FSL SP-058, which is related to the isolated phages MOE1 and MOE2, is a member of the genus Sp58virus [48]. Wittmann et al. argued that the N4-like phages belong to a higher taxonomic division than a genus or subfamily [18]. This is also strengthened by dotplot analysis of the N4-like MCP sequences, which resulted in more subgroups than did comparisons of the other MCP-sequences. Thus, our N4 MCP PCR amplifies viruses of more than one genus and is specific for the group formerly known as "N4-like phages" infecting gammaproteobacteria [38].

The genus "GJ1virus" has not yet been established. The five phages used for the design of the GJ1 MCP primers share $\geq 40 \%$ protein homologs with $\Phi E c o M-G J 1$ as calculated by CoreGenes 3.5 [54], i.e., they fulfill an important criterion that was used in the past to group phages into the same genus [10]. However, the number of homologous proteins using $\Phi$ EcoM-GJ1 as reference varies considerably (PM1, 81\%; Y2, 59\%; Spp001, 48\%; pAh6-C, 40\%), and dotplot analysis of MCP sequences revealed subclusters. Thus, more sequences of GJ1-like phages are required to clarify the taxonomy of the GJ1-like phages and thereby the specificity of this PCR assay. Erwinia phage QceA2, which was isolated as a presumptive GJ1-like phage, shared the highest amino acid sequence similarity with the MCP of Erwinia phage vB_EamM-Y2, which is closely related to phage $\Phi$ EcoM-GJ1 $[11,17]$.

The group of FO1-like phages was reassorted in the past. All phages used for primer design are members of the subfamily Ounavirinae, which was recently established [12]. Related phages of the genera Pakpunavirus and KPP10virus and the proposed genus "KILvirus" infect Pseudomonas strains only $[34,55]$. A BLAST-search of the FO1-primers against the MCP sequences of the PAK_P1-, KPP10-, and KIL-like clades was negative. We thus expect that the FO1-primers only amplify MCP genes of FO1 like phages infecting enterobacteria. Hence, the FO1 MCP PCR specifically identifies members of the subfamily Ounavirinae. This was further supported by partial MCP sequences of 
the presumptive FO1-like isolates PGP, VNV, and QceB10. PGP and VNV were found to be most similar to Salmonella phage UAB_Phi87, and to Escherichia phage vB_EcoMVpaE1, respectively, both of which are members of the genus Felixolvirus [12, 49, 50]. Erwinia phage vB_EamMM7, the closest relative of QceB10, belongs to the genus Ea214virus [12].

Finally, the Vi1 MCP primers were originally designed based on phages of a single genus [29]. A recent reclassification has placed them into two separate subfamilies of a novel family [56]. Thus, the Vi1 MCP PCR selects members of the new family Ackermannviridae. The isolate DaiSi was found to be related to Salmonella phage FSL SP-029, a Vi1-like phage [48].

MCP-PCR-based detection of phages can most probably be adapted to any phage genus or subfamily of interest, provided that sufficient sequence data are available. Consequently, the MCP PCR assay is not suited to identify phages of uncharacterized groups. Despite its rather limited detection range, the MCP PCR assay still allows the discovery of novel phages, because two phages of the same genus do not necessarily share all properties, e.g., host ranges within the target species can differ considerably if two isolates exhibit different tail structures. For example, there is a high variability in genes showing similarities to tail spikes in the N4-like phages, which might be responsible for differences in host specificity [18]. Likewise, the genomes of many podoviruses infecting members of the genus Acinetobacter exhibit high homology and collinearity except for a pectate lyase domain located in their tail fibers, which is responsible for differences in host range [57]. Vi1-like phages also exhibit significant divergence only in their tail spike regions [29]. Depending on their tail structures, Vi1-like phages with high specificity for certain serotypes as well as those infecting members of different genera have been described [58-62]. Finally, the T4-like Salmonella phage S16 which has an extraordinarily broad host range, is characterized by long tail fibers with an unexpected architecture known from T2-like phages [27].

Considering the enormous diversity of bacteriophages in nature, it is very challenging to specifically isolate a phage that perfectly meets all requirements for biocontrol purposes. Thus, an MCP-PCR-based preselection at the very beginning of the isolation process clearly facilitates the search for novel biocontrol phages. Once selected for further applications, the complete phage genome needs to be sequenced to confirm the preliminary classification and to ultimately prove the strictly lytic lifestyle. This also enables identification of potential toxin genes. In addition, the transduction potential of the phage needs to be addressed. Altogether, we conclude that the MCP PCR is a useful method for targeted isolation of selected virulent phages and sorting out of undesired isolates, such as frequently found temperate phages.
Acknowledgements We thank Margarita Salas (Universidad Autónoma de Madrid, Spain) for providing phage \$29, Rob Lavigne (KU Leuven, Belgium) for LBL3, Elisabeth Haggård-Ljungquist (Stockholm University, Sweden) for P2, and Max Schobert (TU Braunschweig, Germany) for JG004

\section{Compliance with ethical standards}

Funding LE.K. and Y.B. were funded by Swiss National Science Foundation (SNF) grant 310030_156947. JK was funded by ETH Zurich PA2070-107.

Conflict of interest The authors declare that they have no conflict of interest.

Research involving human participants and animals Human participants and animals were not involved in this research.

Ethical standards This research complies with the ethical standards of "Archives of Virology".

OpenAccess This article is distributed under the terms of the Creative Commons Attribution 4.0 International License (http://creativeco mmons.org/licenses/by/4.0/), which permits unrestricted use, distribution, and reproduction in any medium, provided you give appropriate credit to the original author(s) and the source, provide a link to the Creative Commons license, and indicate if changes were made.

\section{References}

1. Loc-Carrillo C, Abedon ST (2011) Pros and cons of phage therapy. Bacteriophage 1:111-114. https://doi.org/10.4161/bact.1.2.14590

2. Endersen L, O'Mahony J, Hill C et al (2014) Phage therapy in the food industry. Annu Rev Food Sci Technol 5:327-349. https://doi. org/10.1146/annurev-food-030713-092415

3. Buttimer C, McAuliffe O, Ross RP et al (2017) Bacteriophages and bacterial plant diseases. Front Microbiol 8:34. https://doi. org/10.3389/fmicb.2017.00034

4. Richards GP (2014) Bacteriophage remediation of bacterial pathogens in aquaculture: a review of the technology. Bacteriophage 4:e975540. https://doi.org/10.4161/21597081.2014.975540

5. Hatfull GF (2008) Bacteriophage genomics. Curr Opin Microbiol 11:447-453. https://doi.org/10.1016/j.mib.2008.09.004

6. Cobián Güemes AG, Youle M, Cantú VA et al (2016) Viruses as winners in the game of life. Annu Rev Virol 3:197-214. https:// doi.org/10.1146/annurev-virology-100114-054952

7. Ross A, Ward S, Hyman P (2016) More is better: selecting for broad host range bacteriophages. Front Microbiol 7:1352. https ://doi.org/10.3389/fmicb.2016.01352

8. Adams MH (1959) Bacteriophages. Interscience Publishers Inc., New York

9. Yu P, Mathieu J, Li M et al (2015) Isolation of polyvalent bacteriophages by sequential multiple-host approaches. Appl Environ Microbiol 82:808-815. https://doi.org/10.1128/AEM.02382-15

10. Krupovic M, Dutilh BE, Adriaenssens EM et al (2016) Taxonomy of prokaryotic viruses: update from the ICTV bacterial and archaeal viruses subcommittee. Arch Virol 161:1095-1099. https ://doi.org/10.1007/s00705-015-2728-0

11. Grose JH, Casjens SR (2014) Understanding the enormous diversity of bacteriophages: the tailed phages that infect the bacterial 
family Enterobacteriaceae. Virology 468-470:421-443. https:// doi.org/10.1016/j.virol.2014.08.024

12. Adams MJ, Elliot Lefkowitz BJ, Andrew Q, King BM et al (2017) Changes to taxonomy and the International Code of Virus Classification and Nomenclature ratified by the International Committee on Taxonomy of Viruses (2017). Arch Virol 162:2505-2538. https ://doi.org/10.1007/s00705-017-3358-5

13. Yeh Y, Purushothaman P, Gupta N et al (2017) Bacteriophage application on red meats and poultry: effects on Salmonella population in final ground products. Meat Sci 127:30-34. https://doi. org/10.1016/j.meatsci.2017.01.001

14. Tolen TN, Xie Y, Hernandez AC, Kuty Everett GF (2015) Complete genome sequence of Salmonella enterica serovar Typhimurium myophage Mushroom. Genome Announc 3:e00154-15. https ://doi.org/10.1128/genomeA.00154-15

15. Jamalludeen N, Kropinski AM, Johnson RP et al (2008) Complete genomic sequence of bacteriophage $\Phi$ EcoM-GJ1, a novel phage that has myovirus morphology and a podovirus-like RNA polymerase. Appl Environ Microbiol 74:516-525. https://doi. org/10.1128/AEM.00990-07

16. Lim J-A, Shin H, Lee DH et al (2014) Complete genome sequence of the Pectobacterium carotovorum subsp. carotovorum virulent bacteriophage PM1. Arch Virol 159:2185-2187. https://doi. org/10.1007/s00705-014-2005-7

17. Born Y, Fieseler L, Marazzi J et al (2011) Novel virulent and broad-host-range Erwinia amylovora bacteriophages reveal a high degree of mosaicism and a relationship to Enterobacteriaceae phages. Appl Environ Microbiol 77:5945-5954. https://doi. org/10.1128/AEM.03022-10

18. Wittmann J, Klumpp J, Moreno Switt AI et al (2015) Taxonomic reassessment of N4-like viruses using comparative genomics and proteomics suggests a new subfamily_- Enquartavirinae". Arch Virol 160:3053-3062. https://doi.org/10.1007/s00705-015-2609-6

19. Lau GL, Sieo CC, Tan WS et al (2010) Efficacy of a bacteriophage isolated from chickens as a therapeutic agent for colibacillosis in broiler chickens. Poult Sci 89:2589-2596. https://doi.org/10.3382/ ps.2010-00904

20. Lavigne R, Seto D, Mahadevan P et al (2008) Unifying classical and molecular taxonomic classification: analysis of the Podoviridae using BLASTP-based tools. Res Microbiol 159:406-414. https://doi.org/10.1016/j.resmic.2008.03.005

21. Spricigo DA, Bardina C, Cortés P, Llagostera M (2013) Use of a bacteriophage cocktail to control Salmonella in food and the food industry. Int J Food Microbiol 165:169-174. https://doi. org/10.1016/j.ijfoodmicro.2013.05.009

22. Cornelissen A, Ceyssens PJ, Krylov VN et al (2012) Identification of EPS-degrading activity within the tail spikes of the novel Pseudomonas putida phage AF. Virology 434:251-256. https:// doi.org/10.1016/j.virol.2012.09.030

23. Born Y, Fieseler L, Klumpp J et al (2014) The tail-associated depolymerase of Erwinia amylovora phage L1 mediates host cell adsorption and enzymatic capsule removal, which can enhance infection by other phage. Environ Microbiol 16:2168-2180. https ://doi.org/10.1111/1462-2920.12212

24. Latka A, Maciejewska B, Majkowska-Skrobek G et al (2017) Bacteriophage-encoded virion-associated enzymes to overcome the carbohydrate barriers during the infection process. Appl Microbiol Biotechnol 101:3103-3119. https://doi.org/10.1007/s0025 3-017-8224-6

25. Sarker SA, Berger B, Deng Y et al (2017) Oral application of Escherichia coli bacteriophage: safety tests in healthy and diarrheal children from Bangladesh. Environ Microbiol 19:237-250. https://doi.org/10.1111/1462-2920.13574

26. Sarker SA, McCallin S, Barretto C et al (2012) Oral T4-like phage cocktail application to healthy adult volunteers from
Bangladesh. Virology 434:222-232. https://doi.org/10.1016/j. virol.2012.09.002

27. Marti R, Zurfluh K, Hagens S et al (2013) Long tail fibres of the novel broad-host-range T-even bacteriophage S16 specifically recognize Salmonella OmpC. Mol Microbiol 87:818-834. https ://doi.org/10.1111/mmi.12134

28. Adriaenssens EM, Wittmann J, Kuhn JH et al (2018) Taxonomy of prokaryotic viruses: 2017 update from the ICTV Bacterial and Archaeal Viruses Subcommittee. Arch Virol 163:1125-1129. https://doi.org/10.1007/s00705-018-3723-z

29. Adriaenssens EM, Ackermann H-W, Anany $\mathrm{H}$ et al (2012) A suggested new bacteriophage genus: "Viunalikevirus". Arch Virol 157:2035-2046. https://doi.org/10.1007/s00705-012-1360-5

30. Adriaenssens EM, Van Vaerenbergh J, Vandenheuvel D et al (2012) T4-related bacteriophage LIMEstone isolates for the control of soft rot on potato caused by 'Dickeya solani'. PLoS One 7:e33227. https://doi.org/10.1371/journal.pone.0033227

31. Carter CD, Parks A, Abuladze T et al (2012) Bacteriophage cocktail significantly reduces Escherichia coli O157. Bacteriophage 2:178-185. https://doi.org/10.4161/bact.22825

32. Abuladze T, Li M, Menetrez MY et al (2008) Bacteriophages reduce experimental contamination of hard surfaces, tomato, spinach, broccoli, and ground beef by Escherichia coli $\mathrm{O} 157: \mathrm{H7}$. Appl Environ Microbiol 74:6230-6238. https://doi.org/10.1128/ AEM.01465-08

33. Matilla MA, Fang X, Salmond GPC (2014) Viunalikeviruses are environmentally common agents of horizontal gene transfer in pathogens and biocontrol bacteria. ISME J 8:2143-2147. https:// doi.org/10.1038/ismej.2014.150

34. Henry M, Bobay L-M, Chevallereau A et al (2015) The search for therapeutic bacteriophages uncovers one new subfamily and two new genera of Pseudomonas-infecting Myoviridae. PLoS One 10:e0117163. https://doi.org/10.1371/journal.pone.0117163

35. Dekel-Bird NP, Avrani S, Sabehi G et al (2013) Diversity and evolutionary relationships of T7-like podoviruses infecting marine cyanobacteria. Environ Microbiol 15:1476-1491. https://doi. org/10.1111/1462-2920.12103

36. Smith KC, Castro-Nallar E, Fisher JN et al (2013) Phage cluster relationships identified through single gene analysis. BMC Genomics 14:410. https://doi.org/10.1186/1471-2164-14-410

37. Rose TM, Henikoff JG, Henikoff S (2003) CODEHOP (COnsensus-DEgenerate Hybrid Oligonucleotide Primer) PCR primer design. Nucleic Acids Res 31:3763-3766

38. Chan JZ-M, Millard AD, Mann NH, Schäfer H (2014) Comparative genomics defines the core genome of the growing N4-like phage genus and identifies N4-like Roseophage specific genes. Front Microbiol 5:506. https://doi.org/10.3389/fmicb.2014.00506

39. Han F, Li M, Lin H et al (2014) The novel Shewanella putrefaciens-infecting bacteriophage Spp001: genome sequence and lytic enzymes. J Ind Microbiol Biotechnol 41:1017-1026. https://doi. org/10.1007/s10295-014-1438-Z

40. Jun JW, Kim HJ, Yun SK et al (2015) Genomic structure of the Aeromonas bacteriophage pAh6-C and its comparative genomic analysis. Arch Virol 160:561-564. https://doi.org/10.1007/s0070 5-014-2221-1

41. Krumsiek J, Arnold R, Rattei T (2007) Gepard: a rapid and sensitive tool for creating dotplots on genome scale. Bioinformatics 23:1026-1028. https://doi.org/10.1093/bioinformatics/btm039

42. Knecht LE, Born Y, Pothier JF et al (2018) Complete genome sequences of Erwinia amylovora phages vB_EamP-S2 and vB_ EamM-Bue1. Microbiol Resour Announc 7:e00891-18. https:// doi.org/10.1128/MRA.00891-18

43. Sambrook J, Russell DW (2001) Molecular cloning. Cold Spring Harbor Laboratory Press, Cold Spring Harbor

44. Klumpp J, Dorscht J, Lurz R et al (2008) The terminally redundant, nonpermuted genome of Listeria bacteriophage A511: a 
model for the SPO1-like myoviruses of Gram-positive bacteria. J Bacteriol 190:5753-5765. https://doi.org/10.1128/JB.00461-08

45. Altschul SF, Gish W, Miller W et al (1990) Basic local alignment search tool. J Mol Biol 215:403-410

46. Kaliniene L, Zajančkauskaitè A, Šimoliūnas E et al (2015) Lowtemperature bacterial viruses VR-a small but diverse group of $E$. coli phages. Arch Virol 160:1367-1370. https://doi.org/10.1007/ s00705-015-2388-0

47. Dufour N, Clermont O, La Combe B et al (2016) Bacteriophage LM33_P1, a fast-acting weapon against the pandemic ST131O25b:H4 Escherichia coli clonal complex. J Antimicrob Chemother 71:3072-3080. https://doi.org/10.1093/jac/dkw253

48. Moreno Switt AI, Orsi RH, den Bakker HC et al (2013) Genomic characterization provides new insight into Salmonella phage diversity. BMC Genomics 14:481. https://doi. org/10.1186/1471-2164-14-481

49. Bardina C, Colom J, Spricigo DA et al (2016) Genomics of three new bacteriophages useful in the biocontrol of Salmonella. Front Microbiol 7:545. https://doi.org/10.3389/fmicb.2016.00545

50. Šimoliūnas E, Vilkaitytė M, Kaliniene L et al (2015) Incomplete LPS core-specific Felix01-like virus vB_EcoM_VpaE1. Viruses 7:6163-6181. https://doi.org/10.3390/v7122932

51. Hatfull GF, Hendrix RW (2011) Bacteriophages and their genomes. Curr Opin Virol 1:298-303. https://doi.org/10.1016/j. coviro.2011.06.009

52. Barylski J, Enault F, Dutilh BE et al (2018) Analysis of spounaviruses as a case study for the overdue reclassification of tailed bacteriophages. bioRxiv. https://doi.org/10.1101/220434

53. Adriaenssens EM, Krupovic M, Knezevic P et al (2016) Taxonomy of prokaryotic viruses: 2016 update from the ICTV bacterial and archaeal viruses subcommittee. Arch Virol 162:1153-1157. https://doi.org/10.1007/s00705-016-3173-4

54. Turner D, Reynolds D, Seto D et al (2013) CoreGenes35: a webserver for the determination of core genes from sets of viral and small bacterial genomes. BMC Res Notes 6:140. https://doi. org/10.1186/1756-0500-6-140

55. Rombouts S, Volckaert A, Venneman S et al (2016) Characterization of novel bacteriophages for biocontrol of bacterial blight in leek caused by Pseudomonas syringae pv. porri. Front Microbiol 7:279. https://doi.org/10.3389/fmicb.2016.00279

56. Adriaenssens EM, Wittmann J, Kuhn JH et al (2018) Taxonomy of prokaryotic viruses: 2017 update from the ICTV Bacterial and Archaeal Viruses Subcommittee. Arch Virol. https://doi. org/10.1007/s00705-018-3723-Z

57. Oliveira H, Costa AR, Konstantinides N et al (2017) Ability of phages to infect Acinetobacter calcoaceticus-Acinetobacter baumannii complex species through acquisition of different pectate lyase depolymerase domains. Environ Microbiol 19:5060-5077. https://doi.org/10.1111/1462-2920.13970

58. Kutter EM, Skutt-Kakaria K, Blasdel B et al (2011) Characterization of a ViI-like phage specific to Escherichia coli O157:H7. Virol J 8:430. https://doi.org/10.1186/1743-422X-8-430

59. Shahrbabak SS, Khodabandehlou Z, Shahverdi AR et al (2013) Isolation, characterization and complete genome sequence of PhaxI: a phage of Escherichia coli O157: H7. Microbiology 159:1629-1638. https://doi.org/10.1099/mic.0.063776-0

60. Park M, Lee J-H, Shin H et al (2012) Characterization and comparative genomic analysis of a novel bacteriophage, SFP10, simultaneously inhibiting both Salmonella enterica and Escherichia coli O157:H7. Appl Environ Microbiol 78:58-69. https://doi. org/10.1128/AEM.06231-11

61. Lagonenko AL, Sadovskaya O, Valentovich LN, Evtushenkov AN (2015) Characterization of a new ViI-like Erwinia amylovora bacteriophage phiEa2809. FEMS Microbiol Lett 362:fnv031. https:// doi.org/10.1093/femsle/fnv031

62. Matilla MA, Salmond GPC (2014) Bacteriophage фMAM1, a Viunalikevirus, is a broad-host-range, high-efficiency generalized transducer that infects environmental and clinical isolates of the enterobacterial genera Serratia and Kluyvera. Appl Environ Microbiol 80:6446-6457. https://doi.org/10.1128/AEM.01546-14

63. Salas M (2012) My life with bacteriophage $\varphi 29$. J Biol Chem 287:44568-44579. https://doi.org/10.1074/jbc.X112.433458

64. Garbe J, Bunk B, Rohde M, Schobert M (2011) Sequencing and characterization of Pseudomonas aeruginosa phage JG004. BMC Microbiol 11:102. https://doi.org/10.1186/1471-2180-11-102

65. Ceyssens P-J, Miroshnikov K, Mattheus W et al (2009) Comparative analysis of the widespread and conserved PB1-like viruses infecting Pseudomonas aeruginosa. Environ Microbiol 11:28742883. https://doi.org/10.1111/j.1462-2920.2009.02030.x

66. Klumpp J, Lavigne R, Loessner MJ, Ackermann H-W (2010) The SPO1-related bacteriophages. Arch Virol 155:1547-1561. https:// doi.org/10.1007/s00705-010-0783-0

67. Carlton RM, Noordman WH, Biswas B et al (2005) Bacteriophage P100 for control of Listeria monocytogenes in foods: genome sequence, bioinformatic analyses, oral toxicity study, and application. Regul Toxicol Pharmacol 43:301-312. https://doi. org/10.1016/j.yrtph.2005.08.005

68. O'Flaherty S, Coffey A, Edwards R et al (2004) Genome of staphylococcal phage K: a new lineage of Myoviridae infecting grampositive bacteria with a low G+C content. J Bacteriol 186:28622871. https://doi.org/10.1128/JB.186.9.2862-2871.2004

69. Gill JJ (2014) Revised genome sequence of Staphylococcus aureus bacteriophage K. Genome Announc 2:e01173-13. https://doi. org/10.1128/genomeA.01173-13

70. Nilsson AS, Haggård-Ljungquist E (2007) Evolution of P2-like phages and their impact on bacterial evolution. Res Microbiol 158:311-317. https://doi.org/10.1016/J.RESMIC.2007.02.004

71. Dorscht J, Klumpp J, Bielmann R et al (2009) Comparative genome analysis of Listeria bacteriophages reveals extensive mosaicism, programmed translational frameshifting, and a novel prophage insertion site. J Bacteriol 191:7206-7215. https://doi. org/10.1128/JB.01041-09

72. Schmuki MM, Erne D, Loessner MJ, Klumpp J (2012) Bacteriophage P70: unique morphology and unrelatedness to other Listeria bacteriophages. J Virol 86:13099-13102. https://doi.org/10.1128/ JVI.02350-12

73. Loessner MJ, Inman RB, Lauer P, Calendar R (2000) Complete nucleotide sequence, molecular analysis and genome structure of bacteriophage A118 of Listeria monocytogenes: implications for phage evolution. Mol Microbiol 35:324-340. https://doi.org/10.1 046/j.1365-2958.2000.01720.x

Publisher's Note Springer Nature remains neutral with regard to jurisdictional claims in published maps and institutional affiliations. 